\title{
Le bilan biologique en odontostomatologie : intérêt et interprétation
}

\section{RÉSUMÉ}

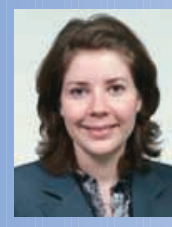

Mariam RERHRHAYE

Résidante au service de parodontologie, C.C.T.D. Rabat,

19 , rue Oued Ziz, appartement 3 ,

Agdal,

Rabat, Maroc.

\section{Lamia ABDELLAOUI}

Professeur assistante en parodontologie, Faculté de médecine dentaire,

de Rabat.

Amal BOUZIANE

Spécialiste en parodontologie, C.C.T.D. Rabat.

\section{Oumkaltoum ENNIBI}

Professeur de l'enseignement supérieur, Chef de service de parodontologie, C.C.T.D. Rabat.
En odontostomatologie, certains actes thérapeutiques nécessitent la réalisation de tests biologiques en préopératoire. La connaissance de ces tests est indispensable pour les prescrire de manière pertinente et pour interpréter les résultats en collaboration avec le médecin traitant. Elle permet d'une part, une bonne planification des soins potentiellement à risque et d'autre part le dépistage précoce de certaines pathologies à manifestations buccales ainsi que la prévention des complications lors des soins bucco-dentaires.

Dans la première partie de cet article, ces différents tests sont décrits ainsi que les principales étiologies pouvant être à l'origine de résultats significatifs. Dans la deuxième partie, les situations dans lesquelles ces tests seront prescrits sont développées ainsi que leurs interprétations. 


\section{Première partie}

$>$

En pratique quotidienne, l'odontologiste peut se trouver confronté à des patients présentant un risque infectieux ou hémorragique lors des soins. Une démarche diagnostique est donc nécessaire avant la réalisation de tout acte pouvant entraîner un saignement et une bactériémie. Dans certains cas, la réalisation de tests biologiques permet de confirmer ou d'infirmer une suspicion de troubles révélés par l'interrogatoire et l'examen clinique. La connaissance du principe de ces tests est donc indispensable pour les prescrire de manière pertinente et pour pouvoir

\section{Hémogramme}

L'hémogramme est une étude quantitative des éléments figurés du sang. En pratique courante, il comporte une numération globulaire, une formule sanguine (nombre des différents types de globules blancs) et une numération des plaquettes (fig. 1).

\section{Numération globulaire}

La numération globulaire consiste à déterminer le nombre de globules blancs et de globules rouges et fournit d'autres indications sur les globules rouges (tableau I).

L'étude des globules rouges (ou hématies) est basée sur l'analyse de l'hémoglobine ( $\mathrm{Hb})$.

- Toute augmentation du taux d'hémoglobine, oriente le diagnostic vers la polyglobulie qui peut avoir plusieurs causes. En effet, il faut distinguer [1] : interpréter les résultats en collaboration avec le médecin traitant.

En fonction des indications, les tests biologiques les plus souvent demandés comprennent un hémogramme, un bilan d'hémostase ainsi que d'autres examens spécifiques.

Dans la première partie de cet article, nous nous proposons de décrire ces différents tests et les principales étiologies pouvant être à I'origine de résultats significatifs. Dans la deuxième partie, nous développerons les situations dans lesquelles ces tests seront prescrits ainsi que leurs interprétations.

- les polyglobulies secondaires à une hypooxygénation ou anoxie tissulaire (séjour en haute altitude, gros fumeurs, bronchopneumopathies obstructives, cardiopathies congénitales, insuffisance cardiaque), à une intoxication par l'oxyde de carbone, à une tumeur rénale, à un cancer primitif du foie, à un fibrome utérin, à un syndrome de Cushing...

- la polyglobulie primitive de la maladie de Vaquez, syndrome myéloprolifératif dont la cause est inconnue.

- Une diminution d'hémoglobine oriente le diagnostic vers une anémie qui est plus fréquente que la polyglobulie. L'anémie est définie par la baisse du taux d'hémoglobine en dessous de $13 \mathrm{~g} / \mathrm{dL}$ chez l'homme, $12 \mathrm{~g} / \mathrm{dL}$ chez la femme et $11 \mathrm{~g} / \mathrm{dL}$ chez la femme enceinte. En effet, c'est la réduction du taux d'hémoglobine fonctionnelle qui définit 

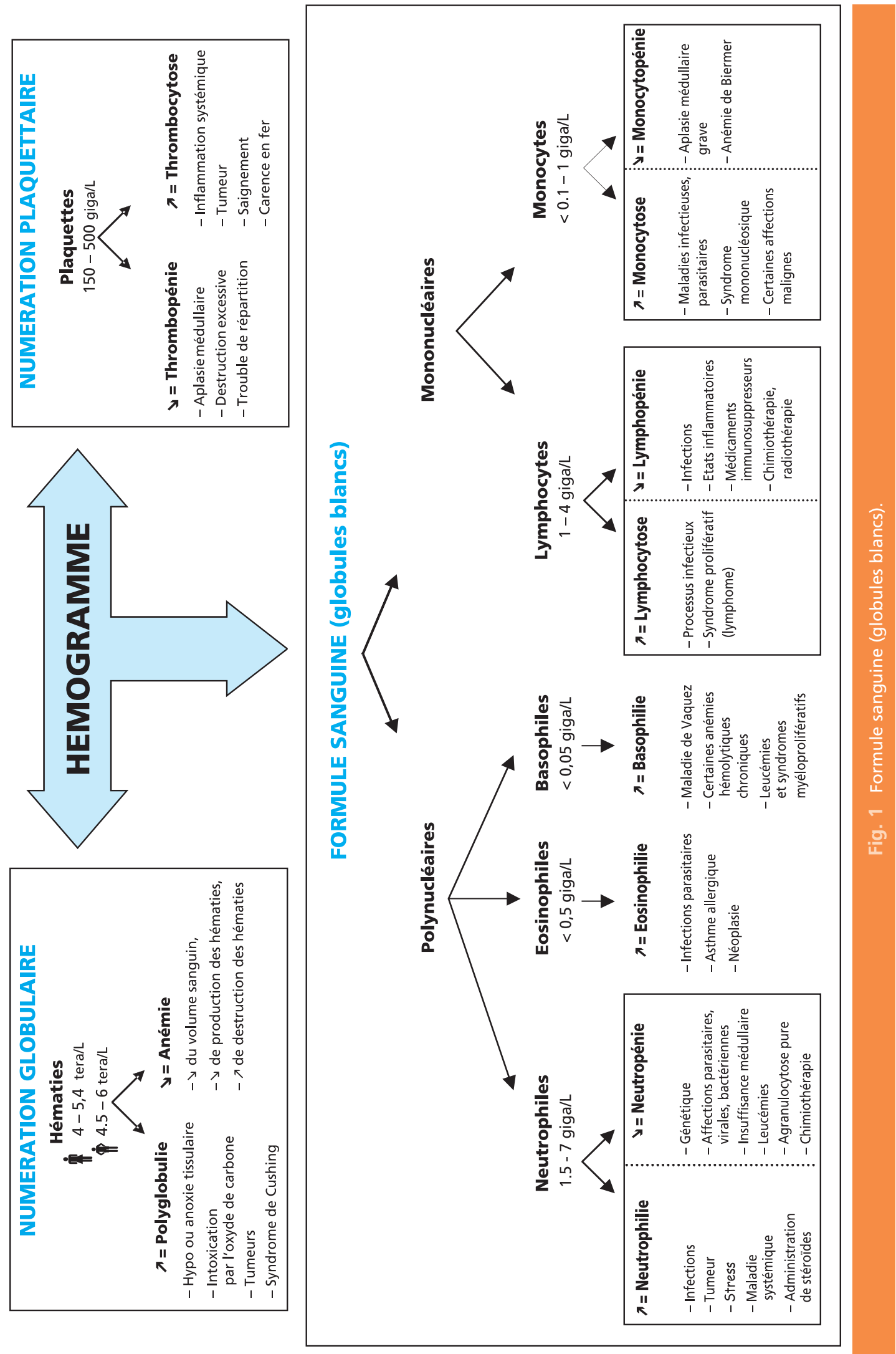
Tableau I

Éléments évalués dans l'hémogramme

\begin{tabular}{|c|c|c|c|c|}
\hline \multirow{2}{*}{$\begin{array}{l}\text { NUMERATION } \\
\text { GLOBULAIRE }\end{array}$} & \multicolumn{2}{|c|}{ Normalité } & \multirow{2}{*}{ Anormalité } & \multirow{2}{*}{$\begin{array}{l}\text { Facteurs de } \\
\text { conversion }\end{array}$} \\
\hline & Homme & Femme & & \\
\hline $\begin{array}{l}\text { Hématies } \\
\text { (tera/L) }\end{array}$ & $4,5-5,9$ & $4-5,4$ & $\begin{array}{l}\boldsymbol{\lambda} \text { : polyglobulie } \\
\boldsymbol{y}: \text { anémie }\end{array}$ & tera $=10^{12}$ \\
\hline $\begin{array}{l}\text { Hémoglobine }(\mathbf{H b})(\mathrm{g} / \mathrm{dL}) \\
\text { mesure photométrique après lyse des GR }\end{array}$ & $13-18$ & $12-16$ & $\begin{array}{l}\boldsymbol{\lambda} \text { : polyglobulie } \\
\boldsymbol{y}: \text { anémie }\end{array}$ & \\
\hline $\begin{array}{l}\text { Hématocrite }(\mathbf{H t}) \\
\begin{array}{c}\text { Volume relatif occupé par les globules rouges dans un } \\
\text { volume donné de sang total }\end{array}\end{array}$ & $0,4-0,54$ & $0,35-0,47$ & $\begin{array}{l}\boldsymbol{\lambda}: \text { polyglobulie } \\
\boldsymbol{У}: \text { anémie }\end{array}$ & 1 unité $=0,01 \%$ \\
\hline $\begin{array}{l}\text { Volume Globulaire Moyen en Hb (VGM) (fL) } \\
\text { (hématocrite/nombre des globules rouges) }\end{array}$ & \multicolumn{2}{|c|}{$\begin{array}{l}80-100 \\
\text { (normocytose) }\end{array}$} & $\begin{array}{l}\boldsymbol{\lambda}: \text { macrocytose } \\
\mathbf{y}: \text { microcytose }\end{array}$ & $\begin{array}{l}1 \text { femtolitre } \\
=10^{-15} \mathrm{~L} \\
=1 \mu \mathrm{m}^{3}\end{array}$ \\
\hline $\begin{array}{c}\text { Concentration corpusculaire Moyenne en Hb } \\
\text { (CCMH) }(\mathrm{g} / \mathrm{dL}) \\
\text { (Hémoglobine/Hématocrite) }\end{array}$ & \multicolumn{2}{|c|}{$31-36$} & $\begin{array}{l}\text { ע : hypochromie } \\
\text { (L'hyperchromie } \\
\text { n'existe pas) }\end{array}$ & $\begin{array}{l}1 \mathrm{~g} / \mathrm{dL} \\
=1 \mathrm{mmol} / \mathrm{L} \times 1,61\end{array}$ \\
\hline $\begin{array}{c}\text { Teneur Corpusculaire Moyenne en Hb (TCMH) } \\
\text { (pg) } \\
\text { (Hémoglobine/nombre des globules rouges) }\end{array}$ & \multicolumn{2}{|c|}{$27-33$} & $\begin{array}{c}\mathbf{y}: \text { hypochromie } \\
\text { (A peu d'usage pratique) }\end{array}$ & $\begin{array}{l}1 \text { picogramme } \\
=10^{-12} \mathrm{~g}\end{array}$ \\
\hline $\begin{array}{c}\text { Réticulocytes (giga/L) } \\
\text { (Hématies jeunes) } \\
\text { (facultative) }\end{array}$ & \multicolumn{2}{|c|}{$20-120$} & $\begin{array}{l}\text { Affirme le caractère } \\
\text { régénératif (origine } \\
\text { périphérique) ou non } \\
\text { (origine centrale) }\end{array}$ & Giga $=10^{9}$ \\
\hline Leucocytes (giga/L) & \multicolumn{2}{|c|}{$\begin{array}{c}4-10 \\
\text { (Variable d'un jour a l'autre, } \\
\nearrow \text { en particulier en période } \\
\text { postprandial, en cas de stress } \\
\text { et d'effort) }\end{array}$} & $\begin{array}{l}\boldsymbol{\lambda}: \text { leucocytose } \\
\boldsymbol{y}: \text { leucopénie }\end{array}$ & \\
\hline
\end{tabular}

I'anémie et non pas la diminution du nombre de globules rouges [2]. L'anémie peut avoir différentes origines: une diminution du volume sanguin, une diminution de la production ou une augmentation de la destruction des globules rouges [3].

Un taux d'hémoglobine inférieur à $10 \mathrm{~g} / \mathrm{dL}$ implique un risque hémorragique. Les actes à l'origine d'un saignement seront donc reportés pour qu'ils aient lieu dans les conditions optimales de sécurité [3].

- Les autres éléments de I'hémogramme (concentration corpusculaire moyenne [CCMH] et volume globulaire moyen en hémoglobine [VGM], réticulocytes) orientent le spécialiste vers le diagnostic étiologique 
de l'anémie ainsi que son traitement (tableau I).

À titre indicatif :

- la CCMH permet de distinguer les anémies hypochromes des anémies normochromes (taille de cellules et concentration d'hémoglobine normale);

- la VGM permet de révéler une microcytose qui confirme l'hypochromie ;

- le taux de réticulocytes affirme le caractère régénératif ou non de l'anémie.

\section{Formule sanguine}

Le deuxième élément de I'hémogramme concerne le nombre de leucocytes qui jouent un rôle fondamental dans la lutte contre l'infection et dans les processus de défense $[3,4,5]$ (fig. 1).

Normalement, le nombre de leucocytes se situe entre 4 et 10 giga/L. Variable d'un jour à l'autre, il augmente particulièrement en période post-prandiale et en cas d'effort ou de stress.

\section{> Les désordres leucocytaires sont classés}

- sur le plan qualitatif en terme d'anomalie fonctionnelle ;

- sur le plan quantitatif en terme d'excès ou d'insuffisance :

- Excès : une augmentation des leucocytes audelà de 10 giga/L caractérise une leucocytose ou hyperleucocytose, d'origine réactionnelle ou maligne par prolifération des cellules de la lignée de la moelle. Il peut s'agir :

- d'une neutrophilie par augmentation du nombre des polynucléaires neutrophiles au dessus de $7 \mathrm{giga} / \mathrm{L}$, résultant le plus souvent d'une infection, d'une tumeur, d'un stress, d'une maladie systémique ou d'une administration de stéroïdes $[3,5,7]$.

- d'une éosinophilie par augmentation du nombre de polynucléaires éosinophiles au dessus de 0,5 giga/L, principalement d'origine allergique, parasitaire ou néoplasique $[3,5]$.

- d'une monocytose, par augmentation du nombre de monocytes au dessus de 1 giga/L, qui se rencontre au cours de l'évolution de nombreuses maladies infectieuses ou parasitaires, d'un syndrome mononucléosique, de certaines affections malignes... $[1,5,6]$;

- d'une lymphocytose, par augmentation du nombre de lymphocytes au dessus de 4 giga/L, qui peut avoir pour origine un processus infectieux ou un syndrome prolifératif (lymphome) $[3,5,6]$;

- d'une basophilie, par augmentation du nombre de polynucléaires basophiles circulants au dessus de 0,05 giga/L. Elle est, exceptionnellement, suffisamment importante pour influer sur le taux des leucocytes. Elle s'observe dans la maladie de Vaquez, certaines anémies hémolytiques chroniques, les leucémies et les syndromes myéloprolifératifs... $[3,5,7]$.

- Insuffisance : une diminution du nombre de globules blancs circulants en dessous de 4 giga/L affirme un diagnostic de leucopénie.

- La leucopénie est due le plus souvent à une réduction du nombre des neutrophiles en dessous de 1,5 giga/L définissant une neutropénie. Lorsque le nombre de neutrophiles est inférieur à 0,5 giga/L, on parle d'agranulocytose $[5,7]$.

La neutropénie peut être d'origine génétique comme la neutropénie familiale et cyclique, ou acquise rencontrée en cas d'affections parasitaires, virales ou bactériennes, de leucémie, d'insuffisance médullaire. Elle peut s'observer 
aussi comme une complication de la chimiothérapie antimitotique $[8,9,10]$.

En général, les personnes avec des déficiences quantitatives et qualitatives des polynucléaires neutrophiles, présentent une destruction sévère des tissus parodontaux [11]. La sévérité des manifestations parodontales est directement liée à la sévérité de la neutropénie [4].

Par ailleurs, toute neutropénie expose à un risque infectieux important ce qui implique des mesures préventives. Une valeur inférieure à 1 giga/L nécessite le report des soins jusqu'à normalisation. En cas d'urgence bucco-dentaire, un traitement en milieu hospitalier est préconisé [3].

- Plus rarement, la baisse porte sur les lymphocytes dont le nombre inférieur à 1 giga/L définit une lymphopénie. Ce déficit peut être ou non associé à un déficit immunitaire qu'il soit primitif ou secondaire à différentes étiologies : infections, états inflammatoires, prise de médicaments immunosuppresseurs, chimiothérapie, radiothérapie...

- La baisse peut porter sur les monocytes, qui, au-dessous de $0.1 \mathrm{giga} / \mathrm{L}$, définissent une monocytopénie, observée dans les aplasies médullaires graves, et l'anémie de Biermer.

\section{Bilan d'hémostase}

L'hémostase est l'ensemble des phénomènes physiologiques qui concourent à l'arrêt du saignement. Elle est classiquement divisée en trois étapes [14, 15] :

- I'hémostase primaire qui délivre le clou plaquettaire. Elle suppose l'interaction des plaquettes avec le sous-endothélium exposé ou une surface endothéliale affectée ;
- Lorsque tous les éléments figurés du sang sont diminués on parle de pancytopénie, pouvant être due à une aplasie médullaire qui correspond à une absence de production des cellules sanguines par la moelle osseuse [12].

\section{Numération plaquettaire}

La numération plaquettaire permet de quantifier le nombre de plaquettes. La normalité est de 150 à 500 giga/L.

Lorsqu'il se produit une diminution du nombre de plaquettes en dessous de 150 giga/L, on parle de thrombopénie. Celle-ci constitue la cause la plus fréquente de saignement anormal. Elle peut être d'origine centrale (insuffisance de production par aplasie ou envahissement médullaire) ou d'origine périphérique (destruction excessive, trouble de répartition) [3].

Une valeur inférieure à 100 giga/L nécessite une transfusion plaquettaire avant la réalisation d'actes saignants [13].

Des chiffres de plaquettes supérieures à 500 giga/L définissent une hyperplaquettose ou thrombocytose. Cette dernière peut être due à une inflammation systémique, une tumeur, un saignement ou une carence en fer $[5,12]$.

- la coagulation qui permet l'obtention du caillot de fibrine, lequel stabilise et solidifie le clou plaquettaire pendant toute la période de cicatrisation de la plaie. Elle se déroule en empruntant 2 voies (fig. 2, tableau II), la voie extrinsèque ou tissulaire (implication des facteurs $\mathrm{V}, \mathrm{VII}, \mathrm{X}$ ) et la voie intrinsèque ou plasmatique (implication des facteurs IX, X, XI, XII). 
Ces deux voies génèrent de la "prothrombinase». A partir de ce complexe enzymatique débute la voie finale commune (facteurs I, II, et XIII) qui permet à la prothrombine d'être transformée en thrombine qui, à son tour, catalyse la transformation du fibrinogène en fibrine.

Chaque voie correspond à une cascade enzymatique, pour laquelle les facteurs de la coagulation, leurs cofacteurs et les membranes cellulaires interagissent en séquences ordonnées. Finalement, les facteurs de coagulation, inertes dans le plasma sous la forme de pro-enzyme, vont être activés tour à tour en enzymes actives.

- La fibrinolyse qui se déclenche en même temps que I'hémostase primaire et la coagulation. Elle élimine le caillot fibrino-plaquettaire afin de rétablir la circulation sanguine et de permettre la
VOIE PLASMATIQUE OU INTRINSEQUE

Surface

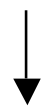

XII, Prékallicréine, kininogène

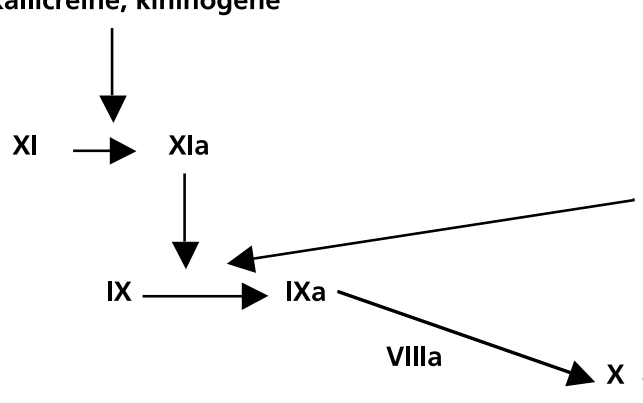

VOIE TISSULAIRE

OU EXTRINSEQUE

Facteur tissulaire + VII

VOIE

FINALE

COMMUNE

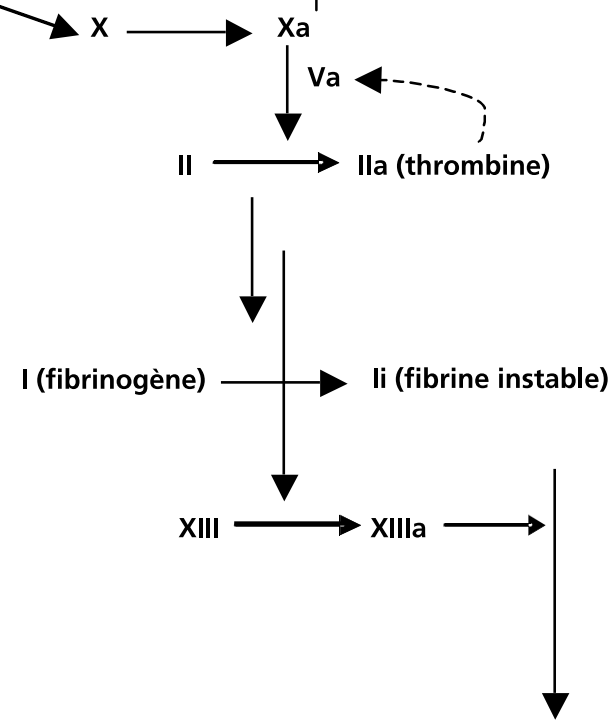

Fibrine stabilisée Is 
Tableau II

Nomenclature internationale définissant les facteurs de coagulation ${ }^{13}$.

\begin{tabular}{ll}
\hline Numéro & Nom \\
\hline I & Fibrinogène \\
\hline II & Prothrombine \\
\hline (III) & Thromboplastine tissulaire (ou cellulaire) facteur tissulaire \\
\hline IV) & (Calcium) \\
\hline VII & Proaccélérine \\
\hline VIII & Proconvertine \\
\hline IX & Facteur antihémophilique A \\
\hline$X$ & Facteur antihémophilique B \\
\hline XI & Facteur Stuart (+) \\
\hline XII & Facteur Hagema Thromboplastin Antécédent) (P.T.A. déficient dans la maladie de Rosenthal) \\
\hline XIII & Facteur stabilisant de la fibrine \\
\hline
\end{tabular}

Les facteurs II, VII, IX, X sont vitamine $K$ dépendants.

Les parenthèses désignent les dénominations non utilisées ; les croix (+) désignent les noms des premiers malades chez lesquels a été caractérisé un déficit en ce facteur.

Remarque : il n'existe pas de facteur IV.

Le numéro d'un facteur suivi d'un «a» signifie que le facteur est activé (pro-enzyme transformé en enzyme).

réparation de la lésion à l'origine de l'hémorragie.

Certains tests de laboratoire sont indispensables à l'évaluation et au diagnostic des troubles de l'hémostase et de la coagulation. On distingue les tests explorant l'hémostase primaire et ceux explorant la coagulation et la fibrinoformation (fig. 3).

\section{Les tests explorant \\ l'hémostase primaire}

\section{$>$ Le temps de saignement (TS) $[3,5,16]$}

Il mesure la durée du saignement provoquée par une incision superficielle [soit au lobe de I'oreille (Duke), soit à l'avant bras (Ivy)].

Les valeurs normales dépendent de la technique utilisée [2 à 4 minutes (Duke), 3 à
5 minutes (Ivy «trois points»), 4 à 8 minutes (Ivy «incision»)]. Le risque hémorragique existe à partir de 10 minutes.

Un allongement du temps de saignement oriente le diagnostic vers deux types de maladies hémorragiques, la thrombopénie (la plus fréquente) et la thrombopathie qui est une anomalie fonctionnelle. Cette dernière est due le plus souvent à certaines médications : acide acétylsalicylique, ticlopidine et autres antiinflammatoires non stéroïdiens. Les hémopathies (syndromes myéloprolifératifs), I'insuffisance rénale chronique, et certaines anomalies constitutionnelles en constituent d'autres causes.

Une numération de plaquettes normale et un temps de saignement normal suffisent à exclure toute anomalie de l'hémostase primaire. 


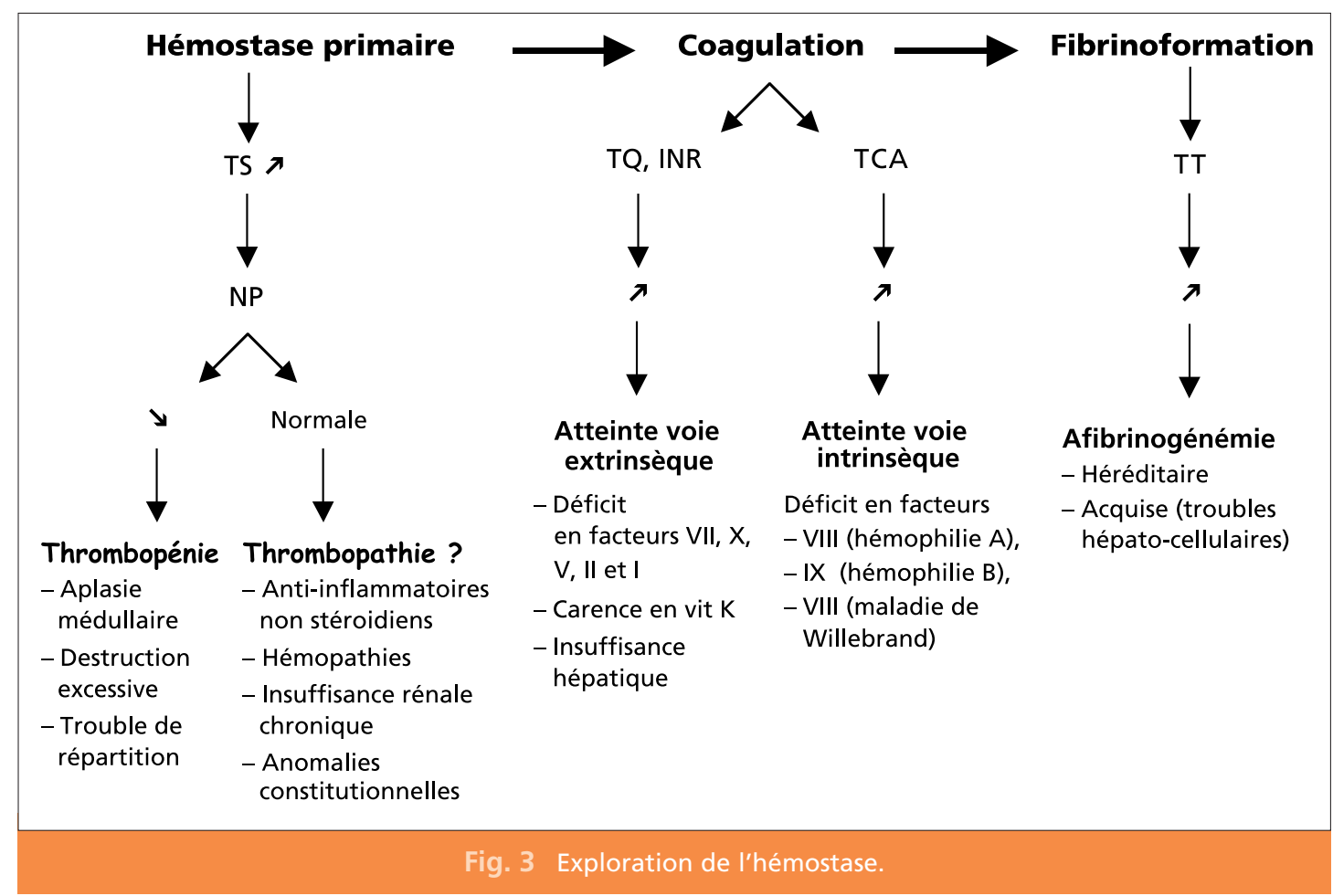

\section{Les tests explorant}

la coagulation

\section{et la fibrinoformation}

\section{> Le temps de Quíck (TQ) ou}

taux de prothrombine (TP) $[5,13,14,16]$

Ce temps évalue la voie extrinsèque de la coagulation. II requiert des concentrations normales de cinq facteurs : VII, X, V, II et I.

Le TQ est normalement compris entre 12 et $14 \mathrm{~s}$, selon les réactifs utilisés. II peut être exprimé en pourcentage par rapport à un témoin, auquel est attribué un taux de $100 \%$. Le TP est normalement supérieur à $70 \%$. En cas d'anomalie, le temps s'allonge et le pourcentage diminue. Un TP inférieur à $40 \%$ comporte un risque hémorragique.

\section{$>$ L'INR (International Normalized Ratio) $[13,14]$}

Il évalue également la voie extrinsèque de la coagulation chez les patients sous traitement par antivitamine K. Il est calculé à partir du temps de Quick (TQ).

INR = (TQ malade/TQ témoin) $x$ Indice de sensibilité international (ISI)

Cet examen donne une interprétation plus facile et plus fiable que le TQ.

- Un INR = 1 est l'équivalent d'un TQ à $100 \%$.

- Un INR > 2 (TP < 40 \%) implique un risque hémorragique.

\section{> Le temps de céphaline} avec activateur ou activé (TCA) [14,16]

Ce temps évalue la voie intrinsèque de la coagulation.

$$
\text { TCA = TCA malade / TCA témoin }
$$

Le TCA est normal quand il est inférieur à 1,2. II indique un risque hémorragique lorsque l'écart est plus de $10 \mathrm{~s}$ par rapport au témoin. 
Ce test explore tous les facteurs de la coagulation, sauf le facteur VII. Les déficits en facteurs VIII et IX correspondent respectivement aux hémophilies $A$ et $B$.

Un déficit généralement modéré en facteur VIII accompagne la maladie de Willebrand. Cette coagulopathie héréditaire résulte de la diminution héréditaire du facteur
Willebrand qui intervient dans l'adhésion primaire des plaquettes au sous-endothélium mis à nu par la lésion vasculaire.

$>$ Le temps de thrombine (TT) $[3,5,14$, II évalue l'ensemble de la fibrinoformation. II mesure le temps de formation du caillot à partir du fibrinogène. Il est anormal quand il dépasse 20 s.

\section{Autres tests d'intérêt clinique en odontostomatologie}

\section{Glycémie}

La glycémie à jeûn est le dosage du taux de glucose dans le sang (valeur ponctuelle). Normalement, elle est comprise entre 3,9 et $5,4 \mathrm{mmol} / \mathrm{L}(0,70$ et $0,95 \mathrm{~g} / \mathrm{L}) \mathrm{chez}$ l'adulte. Le diabète est défini par un niveau de glucose plasmatique à jeun $\geq 7 \mathrm{mmol} / \mathrm{L}$ (1,26 g/L), vérifié à deux reprises [5].

Facteurs de conversion :

$-\mathrm{g} \times 5,56=\mathrm{mmol}$

$-\mathrm{mmol} \times 0,18=\mathrm{g}$

\section{Hémoglobine glyquée} (HbA1c)

C'est une fraction de l'hémoglobine qui stocke le glucose lorsque la glycémie s'élève et dont le taux est fonction des chiffres glycémiques des 2 à 3 mois précédents (la durée de vie des hématies est de 3 mois). Cette mesure permet donc d'évaluer l'équilibre du diabète sur les 2 ou 3 derniers mois. Une HbA1c > $7 \%$ indique un déséquilibre, léger entre 7-8 \%, moyen entre 8-9 \%, et sévère lorsque I'HbA1c est $>9 \%[1,5]$.

\section{Créatinine}

Les valeurs normales dans le sang sont de 80 à $110 \mu \mathrm{mol} / \mathrm{L}$ (9 à $13 \mathrm{mg} / \mathrm{L}$ ) chez l'homme et de 60 à $90 \mu \mathrm{mol} / \mathrm{L}$ (7 à $10 \mathrm{mg} / \mathrm{L})$ chez la femme. Ce dosage est important pour évaluer la clairance et préciser le degré d'une insuffisance rénale chronique (IRC) [5].

Facteurs de conversion :

$-\mathrm{mg} \times 8,8=\mu \mathrm{mol}$

$-\mu \mathrm{mol} \times 0,11=\mathrm{mg}$

\section{Clairance}

\section{de la créatinine endogène}

Les valeurs normales sont comprises entre 75 et $125 \mathrm{ml} /$ minute. Elle baisse en moyenne de $1 \%$ par an à partir de 40 ans.

Cette mesure permet d'estimer le degré d'insuffisance rénale et d'en suivre la progression [5]. 


\begin{tabular}{ll}
\hline Clairance $(\mathrm{ml} / \mathrm{min})$ & Degré d'insuffisance rénale \\
\hline$\leq 60$ & Insuffisance rénale débutante \\
\hline$>30$ & Insuffisance rénale modérée \\
\hline $15-30$ & Insuffisance sévère \\
\hline $10-15$ & Insuffisance rénale nécessitant le recours prochain à la dialyse \\
\hline$<10$ & Nécessité de dialyse \\
\hline
\end{tabular}

\section{Transaminases (SGOT, SGPT)}

En pratique, on évalue l'activité des transaminases glutamo-pyruvique (SGPT) ou asparate aminotransférase (ASAT) et glutamo-oxaloacétique (SGOT) ou alamine aminotransférase (ALAT).

Normalement, la SGOT est de 7 à $40 \mathrm{UI} / \mathrm{l}$ chez I'homme et 5 à $30 \mathrm{UI} / \mathrm{l}$, chez la femme. La SGPT est de 4 à $50 \mathrm{UI} / \mathrm{l}$ chez l'homme et 2 à 40 UI/I chez la femme.

L'augmentation des transaminases témoigne de la cytolyse hépatique, qui peut être très importante dans l'hépatite, surtout virale et médicamenteuse, dans les tumeurs hépatiques et dans l'infarctus du myocarde $[1,17]$.

\section{Bilirubine}

La concentration de la bilirubine plasmatique est de 3 à $12 \mu \mathrm{mol} / \mathrm{L}$, en tout cas inferieure à $18 \mu \mathrm{mol} / \mathrm{L}$ (10 mg/L), elle est exclusivement sous forme non conjuguée. La bilirubine est augmentée en cas de cirrhose hépatique, d'ictère par hépatite, d'ictère mécanique ou d'ictère hémolytique $[1,5]$.

Facteurs de conversions :

$-\mathrm{mg} \times 1,7=\mu \mathrm{mol}$

$-\mu \mathrm{mol} \times 0,58=\mathrm{mg}$.

\section{Vitesse de sédimentation}

La vitesse de sédimentation est un autre bilan biologique qui peut être utile dans le dépis- tage et la surveillance des processus inflammatoires et infectieux malgré son manque de spécificité. Normalement, après une heure, elle est :

\begin{tabular}{ccc}
\hline $\begin{array}{c}\text { Valeurs usuelles } \\
(\mathrm{mm})\end{array}$ & Homme & Femme \\
\hline Jeune & $<15$ & $<20$ \\
\hline 65 ans & $<20$ & $<25$ \\
\hline
\end{tabular}

Dans certains contextes pathologiques, l'élévation reste modérée, le plus souvent inférieure à $50 \mathrm{~mm}$ à la première heure (anémies, obésité, dyslipidémies, insuffisance rénale chronique) [18].

Lorsque la VS est supérieure à $100 \mathrm{~mm}$, il s'agit, dans un cas sur deux, d'une pathologie infectieuse (notamment respiratoire et urinaire), dans $25 \%$ des cas d'une maladie tumorale, et dans les $25 \%$ restants d'une affection inflammatoire [18].

\section{Test ELISA}

\section{(enzyme-linked immunosorbent assay)}

Le test ELISA permet le dépistage d'anticorps VIH. En cas de positivité, ce test doit être confirmé par un test plus spécifique tel que I'analyse par la technique de WesternBlot. 


\section{Sérologie hépatite B}

Le diagnostic de I'hépatite $B(H B)$ repose sur la détection en ELISA d'antigènes viraux ou de leur anticorps, I'apparition des anticorps entraînant la disparition des antigènes.

Le diagnostic virologique direct repose sur la mise en évidence des antigènes du virus dans le sérum du malade. En pratique courante, on recherche l'antigène $\mathrm{HBs}$, antigène de surface constituant l'enveloppe virale, et l'antigène $\mathrm{HBe}$, antigène lié au core, distinct de l'antigène $\mathrm{HBC}$ qui est lié à la nucléocapside.

Le diagnostic virologique indirect repose sur la mise en évidence des anticorps antivirus HB dans le sérum. On peut rechercher les anticorps anti-HBs, anti-HBc et anti-HBe $[5,19]$.

\section{Sérologie hépatite C}

Le diagnostic virologique de I'hépatite $C$ repose sur la mise en évidence des anticorps anti-VHC par un test ELISA. Ce test ne comporte pas de faux négatif chez les patients immunocompétents mais risque de donner de faux positif notamment chez les patients atteints de pathologie auto-immune $[20,21]$.

\section{MNI test}

\section{(mononucléose infectieuse test)}

C'est un examen sérique indirect qui a pour but d'affirmer le diagnostic d'une mononucléose infectieuse. Il est précocement positif, mais il a l'inconvénient de ne pas avoir une spécificité absolue dans tous les cas, c'est pourquoi on l'associera à une réaction sérologique de Paul-Bunnell-Davidson [22].

\section{Réaction sérologique de Paul-Bunnell-Davidson}

Le test se positive à la fin de la première semaine de la mononucléose infectieuse et le reste de trois mois à un an, à des taux compris entre $1 / 160$ et $1 / 1280$ [5].

\section{Bibliographie de la première partie}

1. Girard P, Penne G, Missika P.

Médecine

et chirurgie dentaire.

Problèmes médicaux en pratique quotidienne.

Paris : Éditions CdP, 1988.

2. Benchakroun S.

Les anémies, définition, généralités, classification physiopathologique. Espérance Médicale Mars 2000;7(59):117-122.
3. Roche $\mathrm{Y}$. Chirurgie dentaire et patients à risque. Paris : Flammarion Médecine-Sciences, 1996.

4. Guyton AC. Précis de physiologie médicale. Italie : Piccin, 1996.

5. Caquet $\mathrm{R}$. 250 examens de laboratoire, prescription et interprétation. 10 édition, Masson, 2008.
6. Le Breton $\mathrm{G}$. Traité de sémiologie et clinique odonto-stomatologique. Paris : Éditions CdP, 1997.

7. Trachli A, Madani A, Zafad S, Benchekroun S. L'hémogramme. Espérance Médicale Mars 2000;7(59)123-127.

8. Szpirglas $\mathrm{H}$, Lacoste JP. Manifestations buccales des hémopathies. Encycl Méd Chir 
Stomatologie-odontologie 22-050-A-10, 1994, 8p.

9. Sebahoun $\mathrm{G}$.

Agranulocytoses

iatrogéniques :

étiologies, diagnostic,

traitement.

Rev Prat Paris

1999;49:1355-1360.

10. Szpirglas H, Benslama L. Pathologie

de la muqueuse buccale.

Nancy : Ed Scientifiques

et Médicales Elsevier, 1999.

11. Kinane DF.

Periodontitis modified

by systemic factors.

Ann Periodontol

1999;4(1):54-63.

12. Samson J, Rossier $S$,

Carrel JP.

Hémopathies

et médecine buccale.

Réalités Clin

1999;10(3):407-429.

13. Bouziane $A$, Benrachadi $L$, Ennibi OK, Abdellaoui A, Benzarti N.

Maladies hématologiques : manifestations parodontales et prise en charge.

Rev Odont Stomat

2002;31:299-320.

14. Aillaud MF, Alexandre $P$,

Bierme R.

Hémostase

et thrombose.

4e éd., Ed. Impression

la Simarre, Joué-lès-Tours, 1994.

15. Dridi SM, Wierzba CB,

Meyer J, Pellat B.

Syndromes

hémorragiques :

intérêt clinique

des tests biologiques.

Rev Odont Stomatol

1998;27(3):165-177.

16. Gaussem P, Aiach $M$.

Exploration

de l'hémostase

en biologie clinique.

Actualités Odont Stomat 1996;193:71-77.

17. Mariotti A.

Laboratory testing

of patients

with systemic conditions

in periodontal practice.

Periodont 2000

2004;34:84-108.
18. Cosserat J.

Vitesse de sédimentation

élevée.

In : Traité de Médecine

Akos [1-1450] Paris :

Elsevier Masson SAS, 1998.

19. Girard P, Penne G,

Missika P.

Médecine

et chirurgie dentaire.

Problèmes médicaux

en pratique quotidienne.

Paris : Éditions CdP, 1988.

20. Benazzouz $M$,

Jarroullah MY, Essaid AE.

Hépatite virale $C$.

Rôle du médecin

généraliste.

Rev Prat 2004;XIV(2):27-28.

21. Mrani $S$, Gille $Y$, Baaj $A$.

Biologie moléculaire

et prise en charge

de l'hépatite $C$.

Cah Méd

2003; VI (66):13-15.

22. Girard $\mathrm{P}$, Jeandot $\mathrm{J}$,

Quevauvilliers J,

Perlemuter L.

Dictionnaire médical

du chirurgien-dentiste.

Paris : Éditions Masson, 1997. 


\section{Deuxième partie}

Après avoir décrit dans la première partie de cet article les principaux tests biologiques pouvant présenter une signification clinique en odontostomatologie, nous développerons dans cette deuxième partie, les situations dans lesquelles la prescription de ces tests s'impose ainsi que leur intérêt dans la pratique odontostomatologique.

\section{Quand prescrire? Pourquoi?}

En clinique, il existe trois situations pour lesquelles le praticien peut prescrire des tests biologiques:

- si un trouble est suspecté à la suite de l'interrogatoire médical ou devant des anomalies cutanées ou muqueuses révélées par l'observation clinique (pâleur des muqueuses, pétéchies, ecchymoses, gingivorragies spontanées, infections à répétition) ;

- si un trouble est connu, en situation préopératoire selon les données recueillies au cours de l'interrogatoire et en prévision des risques de l'intervention envisagée (diabète, cardiopathie, antécédents hémorragiques) ;
- en présence de certaines médications qui peuvent avoir des répercussions en per- et post-opératoire comme les anticoagulants (héparine, antivitamine K) et les antiagrégants plaquettaires (aspirine, ticlopidine).

Les résultats de ces tests permettent de confirmer ou d'infirmer une suspicion de trouble révélé par l'interrogatoire et l'examen clinique, et ainsi le dépistage précoce de certaines pathologies à manifestations buccales. De même, ils orientent le praticien vers les précautions à prendre chez les patients présentant une maladie générale connue, permettant ainsi la prévention des complications lors des soins bucco-dentaires [1, 2].

\section{Quels tests prescrire, pour quels patients?}

La prescription des tests, aussi bien de dépistage que de surveillance des maladies systémiques, dépendra des informations obtenues par l'interrogatoire médical et l'observation clinique.

\section{Tests de dépistage}

- Une anémie peut être suspectée devant une pâleur et une atrophie des muqueuses [3] et doit inciter le praticien à prescrire une NFS (fig. 4).

- Devant des ulcérations douloureuses des muqueuses buccales associées à des destructions importantes des tissus parodontaux et une fièvre élevée [4], une neutropénie est suspectée et nécessite aussi la prescription d'une NFS (fig. 5).

- En présence de signes hémorragiques (pétéchies, hématome, gingivorragies), une throm- 

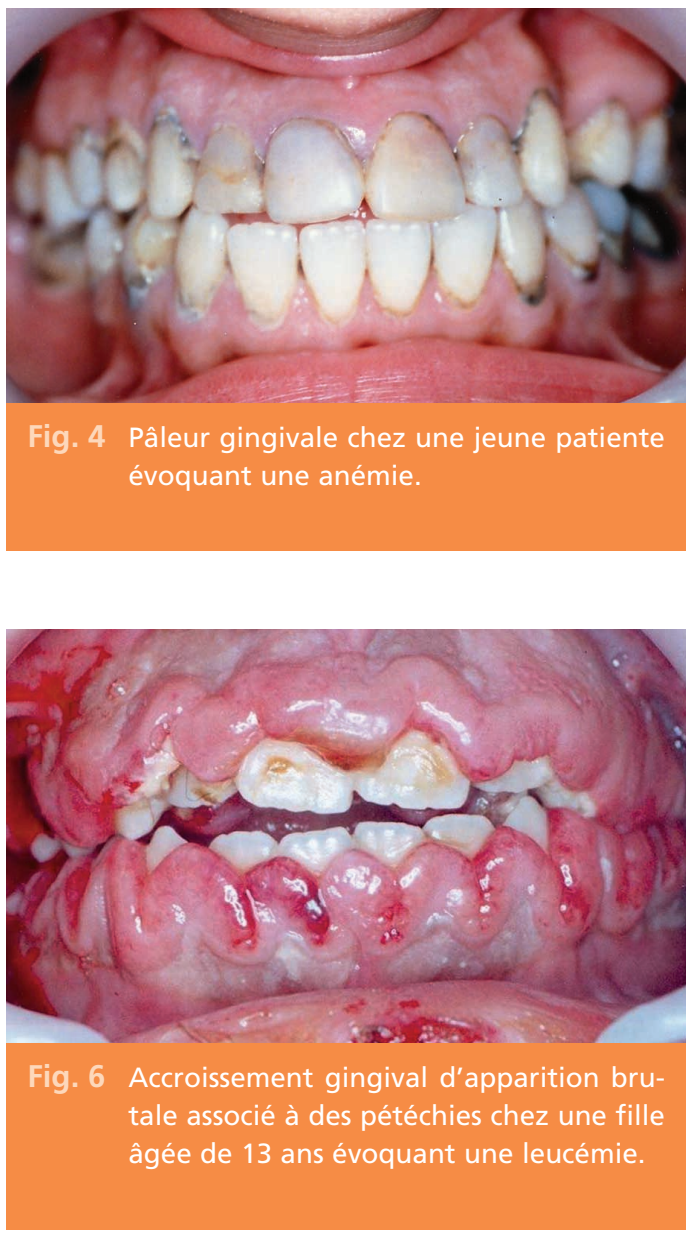

bopénie peut être suspectée, justifiant la prescription d'une numération plaquettaire (NP).

- Si les données recueillies ne suffisent pas à caractériser un trouble hémorragique suspecté, il faut prescrire un bilan d'hémostase : NP, TS, TQ, TCA et TT.

- Une leucémie peut être suspectée devant des manifestations cliniques représentées par une fatigue, une pâleur, une fièvre, des infections traînantes ou à répétition, des syndromes hémorragiques, des polyadénopathies, des douleurs osseuses ainsi que devant certains signes oraux : accroissement gingival, infections et ulcérations des muqueuses. Sur le plan radiographique, des images

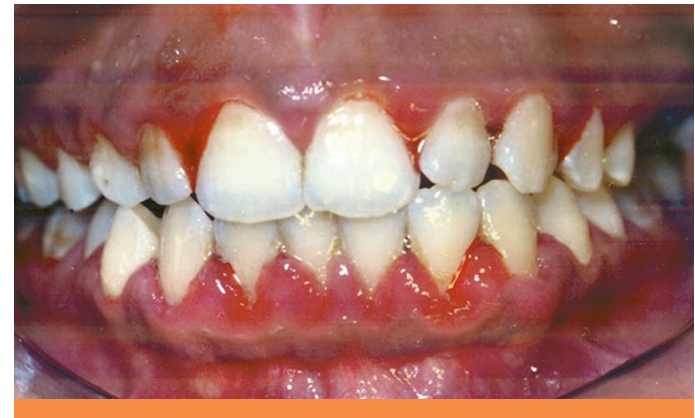

Fig. 5 Ulcérations douloureuses des muqueuses buccales chez une patiente présentant une neutropénie.

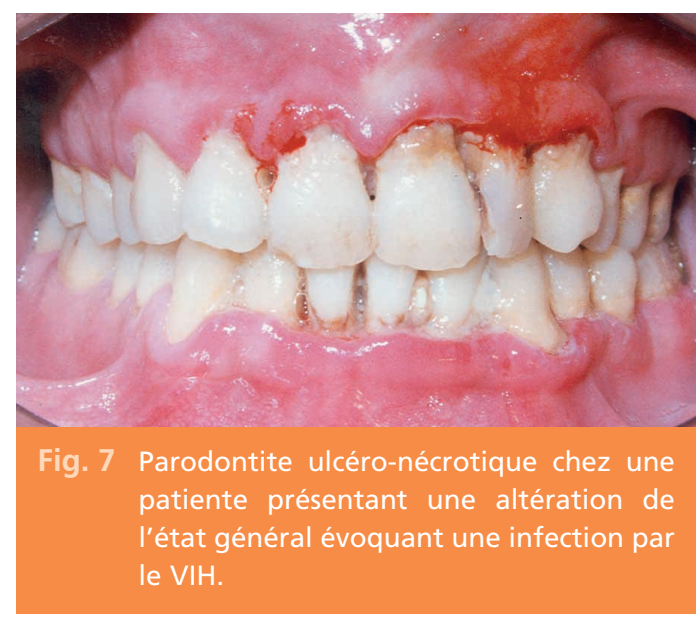

claires avec perte de la lamina dura et érosions des crêtes alvéolaires, peuvent être observées. Le praticien doit prescrire un hémogramme et un TS. En présence de résultats anormaux, le patient sera immédiatement adressé à un hématologue pour évaluation complète et traitement (fig. 6) [1, 5].

- Le diabète est suspecté devant des antécédents familiaux de diabète, des signes généraux (polydipsie, polyurie, polyphagie, perte de poids, obésité, infections cutanées répétitives, troubles de la vision) et des signes oraux (gingivites persistantes, parodontites sévères et actives par rapport à l'âge et à I'hygiène buccale, candidoses, aphtes à répétition, polycaries, sécheresse buccale, retards 

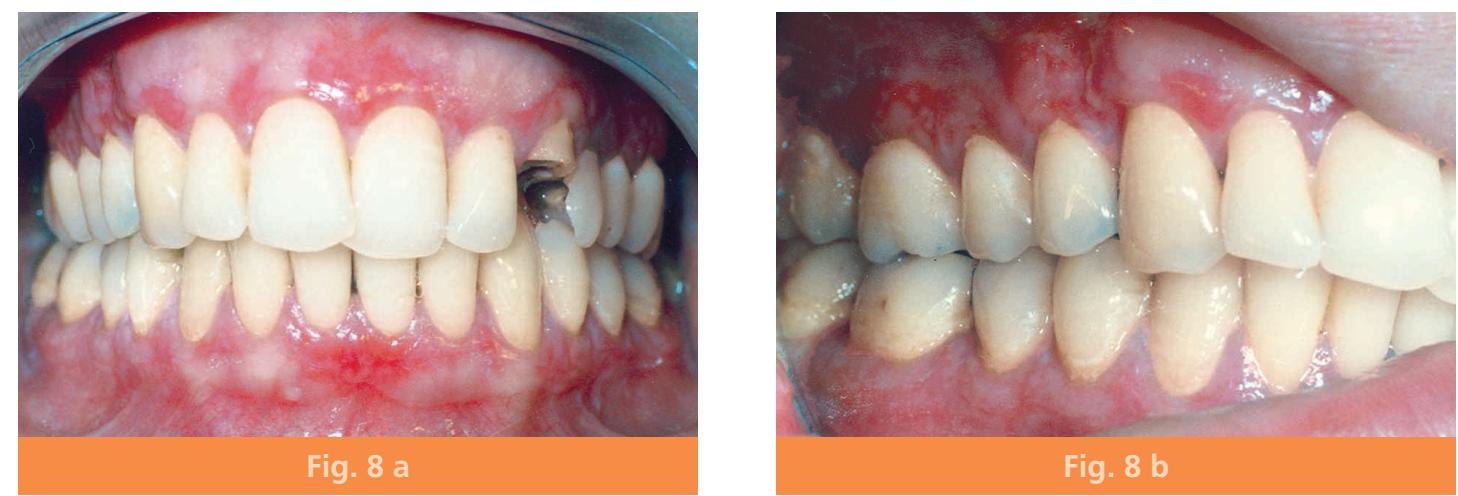

Fig. 8 a et b Lichen plan buccal faisant suspecter une hépatite virale associée.

de cicatrisation, ulcérations notamment après intervention chirurgicale, odeur acétone de l'haleine, hypotonicité de la langue, hyperviscosité salivaire). Face à ce contexte, le praticien doit prescrire un dosage de la glycémie à jeûn $[1,6,7,8,9]$.

- Une infection par le VIH est suspectée en présence de formes atypiques de maladies parodontales comme la gingivite et la parodontite ulcéronécrotique (fig. 7) ou l'érythème gingival linéaire $[10,11,12]$ ou devant des lésions buccales comme une candidose récidivante ou une leucoplasie chevelue $[1,13]$. Le diagnostic immunologique repose sur la recherche d'anticorps VIH sériques par le test de dépistage ELISA et par des tests de confirmation comme le Western-Blot. Le diagnostic hématologique repose sur une lymphopénie majeure (la progression de l'infection HIV vers le SIDA est définie par un taux des lymphocytes $T$ CD4 inférieur à 200/(mm³), une anémie et une thrombopénie, mises en évidence par une NFS $[13,14,15]$.

- Une mononucléose infectieuse peut être suspectée en présence de signes cliniques associant fièvre, asthénie, myalgie, manifestations rhino-pharyngées, adénopathies cervi- cales à des manifestations buccales (pétéchies palatines, ulcérations gingivales) $[5,16]$. Le diagnostic repose sur la NFS qui montre une hyperleucocytose et une monocytose [17].

- Une hépatite virale peut être suspectée en présence d'ictère, de signes hémorragiques ou de lichen plan (fig. 8 a et b). Les anomalies biologiques associent une cytolyse marquée avec élévation des transaminases ASAT et ALAT jusqu'à 20 fois la normale et une hyperbilirubinémie variable avec l'intensité de l'ictère. Le patient sera adressé à un hépatologue $[1,5]$.

\section{Tests de surveillance de maladies systémiques}

- En cas de diabète connu et du fait du risque infectieux encouru, il faut demander un dosage de la glycémie et de l'hémoglobine glyquée pour évaluer l'équilibre du diabète sur les deux ou trois derniers mois [6].

- En cas d'hépatite, le praticien doit prescrire : TS-NP-TQ du fait de la thrombopénie et des troubles de la coagulation en rapport avec les dysfonctions hépatiques, et une NFS du fait de la neutropénie souvent associée à l'hépatite. 
Si le patient a fait une hépatite $A$ et qu'il est guéri, il n'y a aucune précaution particulière à prendre lors des soins.

Si le type d'hépatite est inconnu ou s'il s'agit d'une hépatite $B$, le médecin traitant sera consulté, en particulier pour savoir si l'antigène HBs est présent. Typiquement, il se développe pendant 20 à 200 jours après le contact et reste détectable pendant 120 jours. (Sa persistance au-delà de 13 semaines après la fin de la maladie clinique indique un état porteur persistant.)

L'antigène $\mathrm{HBe}$ sera également recherché car s'il persiste, il indique que la maladie demeure active et à haute contagiosité (au-delà de 4 semaines après l'installation des symptômes le patient restera infectant et développera I'hépatite chronique) $[5,18]$.

- En cas d'atteinte par I'HIV, il faut déterminer le statut hématologique (NFS, TQ, TS) et immunitaire (taux des lymphocytes T CD4). Le stade sida correspond à un taux LT CD4 $<200 / \mathrm{mm}^{3}[1,18]$.

- Si le patient présente une insuffisance rénale et hémodialysé, il faut prescrire une NFS du fait de l'anémie qui accompagne

\section{Conclusion}

La connaissance des tests biologiques est indispensable pour les prescrire de manière pertinente et pour interpréter les résultats en collaboration avec le médecin traitant. Elle permet d'une part, le dépistage précoce de certaines
I'insuffisance rénale, un TS du fait de la thrombopathie et une NP, un TT et un TQ pour évaluer les troubles liés à I'héparinothérapie $[19,20]$. II est important également d'effectuer un dosage de la créatinine pour préciser le degré d'insuffisance rénale et sa répercussion sur le métabolisme des médicaments à élimination rénale, évitant ainsi les prescriptions médicamenteuses inadaptées.

- Si le patient est sous médication chronique à base d'antiagrégant plaquettaire, il faut prescrire un TS avant tout acte saignant.

- Si le patient est traité par des AVK, un INR doit être prescrit pour évaluer le risque hémorragique.

Cette liste n'est pas exhaustive, elle rapporte les principales pathologies rencontrées dans notre pratique quotidienne.

Lorsque les résultats confirment un risque hémorragique ou infectieux lors des soins, le médecin traitant est contacté pour discuter des soins envisagés, des précautions à prendre et du protocole à suivre. II pourra prescrire des tests de deuxième intention et/ou des examens complémentaires.

pathologies à manifestations buccales et d'autre part une bonne planification des soins potentiellement à risque pour une prévention des complications lors des soins bucco-dentaires. 


\section{Bibliographie de la deuxième partie}

1. Roche $Y$.

Chirurgie dentaire

et patients à risque.

Paris : Flammarion

Médecine Sciences, 1996.

2. Dridi $S M$, Wierzba $C B$, Meyer J, Pellat B.

Syndromes

hémorragiques:

intérêt clinique

des tests biologiques.

Rev Odont Stomatol

1998;27(3):165-177.

3. Quessar A, Benchekroun S. L'anémie par carence martiale.

Esperance Médicale 2000;7(59):123-127.

4. Kinane DF.

Periodontitis

modified

by systermic factors.

Ann Periodontol

1999;4(1):54-63.

5. Girard P, Penne G,

Missika P.

Médecine

et chirurgie dentaire.

Problèmes médicaux

en pratique quotidienne.

Paris : Édition Cdp, 1988.

6. Hage G, Davarpanah M,

Tecucianu JF.

Diabète

et état parodontal :

données actuelles.

Journal de Parodontologie

et d'Implantologie Orale

1999;18(1):17-26.

7. Hostetter MK.

Perspectives in diabetes.

Handicaps to host defense.

Effects of hyperglycemia

on C3 and Candidas

albicans.

Diabetes 1990;39:271-275.
8. Gautier D, Bauduceau B, Bellavoir A.

Glandes endocrines

et stomatologie.

EMC 1989, 22050 E 10:9.

Paris : Éditions Techniques.

9. Rose LF, Steinberg BJ,

Atlas SL.

Periodontal

management

of medically

compromised patient.

Periodont 2000

1995;9:165-175.

10. Ryder MI.

An update on HIV

and periodontal

disease.

J Periodont 2002;73:

1071-1078.

11. Holmstrup $P$.

Non plaque induced

gingival lesions.

Ann Periodont 1999;4:20-29.

12. Zamanian $P$, Cuisinier $F$,

Schaaf JF, Tenenbaum $\mathrm{H}$,

Lang JM.

Méthode diagnostique

et prise en charge

thérapeutique

des parodontopathies

associées à l'infection

par le virus VIH.

Inform Dent, Paris

1997;33:2349-2357.

13. Girard P, Jeandot J,

Quevauvilliers J,

Perlemuter L.

Dictionnaire médical

du chirurgien-dentiste.

Paris : Éditions Masson, 1997.

14. Aubert F, Guittard P.

L'essentiel médical

de poche.

Paris : Éditions Marketing /

Ellipses, 1995.
15. Marx RE, Stern D.

Oral and maxillofacial

pathology.

Quintess Books 2003:17-135.

16. Corbet EF

Diagnosis of acute

periodontal lesions.

Periodont 2000

2004;34:204-216.

17. De Labrouhe C, Favre E,

Bertrand JC.

Adénopathies

cervicofaciales.

EMC,

Éditions Scientifiques

et Médicales Elsevier SAS,

Paris, Stomatologie.

22-037-P-10, 2000, 10p.

18. Bouziane A, Benrachadi L, Abdellaoui L, Ennibi OK,

Benzarti N.

Les maladies

infectieuses :

manifestations

buccales,

parodontales

et prise en charge.

Première partie :

maladies virales.

Rev Odont Stomat

2005;34:235-255.

19. Rabai M, Benkirane $M$

Louzi L, Nejmi S.

Les traitements

anticoagulants.

Espérance Médicale

1997;4(34):466-473.

20. Ismaili Z, Lahlou K, Ennibi OK, Benamar L, Benzarti N.

Prise en charge

des patients

hémodialysés

au cabinet dentaire.

Espérance Médicale

1997;4(34):489-493. 


\section{SUMMARY}

\section{Biological tests in dentistry: interest and interpretation}

\section{Mariam RERHRHAYE, Lamia ABDELLAOUI, Amal BOUZIANE, Oumkaltoum ENNIBI \\ Keywords \\ - biological tests \\ - hemogram \\ - hemostasis tests \\ - dentistry}

In dentistry, some therapeutic procedures require biological tests preoperatively. The understanding of these tests is essential for their right prescription and for a good interpretation of the results in collaboration with the physician. It allows a good care planning for potentially procedures of risk, the early detection of pathologies that have oral manifestations and the prevention of complications during dental care.

In the first part of this article, these tests are described and their anomalies explained. In the second part, situations in which these tests will be required are developed and their interpretations analyzed.

\section{Abrégé de psychologie à l'usage des praticiens \\ "Lire entre les dents "}

Cet ouvrage est destiné à former les praticiens sur un champ de connaissances beaucoup trop négligé dans notre profession de santé. En effet, la psychologie est un domaine complexe et seul un odontologiste ayant la double formation pouvait se permettre d'en concevoir les applications spécifiques.

Conçu pièce par pièce le lecteur pourra aborder cet ouvrage au hasard des chapitres qui attirent sa curiosité, mais en se prenant au jeu de la lecture, il pourra se construire une connaissance complète des bases fondamentales en ce qui concerne la coopération thérapeutique.

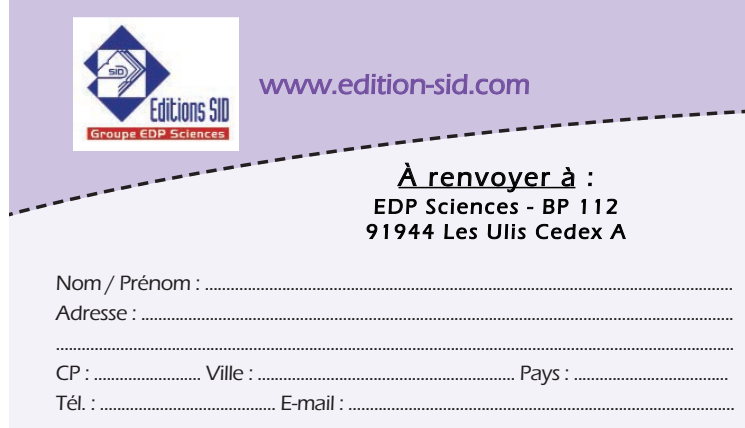

232 pages, noir et blanc format $17 \times 24 \mathrm{~cm}$

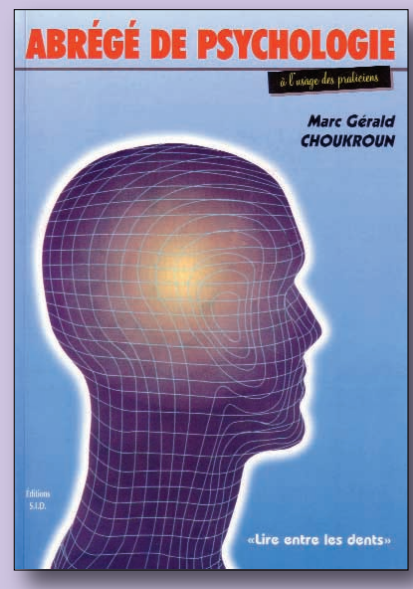
$68 € \mathrm{TTC}$

\begin{tabular}{|l|c|c|c|c|}
\hline \multicolumn{3}{c}{ Titre } & \multicolumn{1}{c}{ Prix } \\
\hline Abrégé de psychologie & & \\
\hline Frais de port & 1 livre & 2 livres & 3 livres & 4 \\
\hline France métropolitaine & $+4 \epsilon$ & $+5 \epsilon$ & $+6 \epsilon$ & $+7 \epsilon$ \\
\hline DOM et Europe & $+7 \epsilon$ & $+9 \epsilon$ & $+11 \epsilon$ & +13 \\
\hline TOM et reste du monde & $+9 \epsilon$ & $+12 \epsilon$ & $+15 \epsilon$ & +18
\end{tabular}

* Aucune commande ne pourra être expédiée sans ajout des frais de port.

Paiement:

$\square$ par chèque à l'ordre d'EDP Sciences (à joindre à la commande) $\square$ par carte bancaire : $\quad \square$ Visa $\quad \square$ Eurocard $\square$ American Express

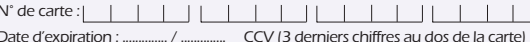

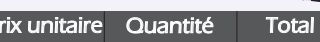

$68 € \quad X$

4 lives 5 livres ou pl......

4 livres 5 livres ou plus

\begin{tabular}{l|l}
$+7 €$ & Gratuit \\
$+13 \epsilon$ &
\end{tabular}

TOTAL GÉNÉRAL

Signature: 